Surgery for

Congenital

Heart Disease

\title{
Left-sided lesions after anatomic repair of transposition of the great arteries, ventricular septal defect, and coarctation: Surgical factors
}

\author{
Siamak Mohammadi, MD, Alain Serraf, MD, PhD, Emre Belli, MD, Bertrand Aupecle, MD, André Capderou, MD, \\ Francois Lacour-Gayet, MD, Ivo Martinovic, MD, Dominique Piot, MD, Anita Touchot, MD, Jean Losay, MD, and \\ Claude Planché, MD
}

From the Department of Pediatric Cardiac Surgery, Marie-Lannelongue Hospital, Le Plessis-Robinson, France.

Read at the Eighty-third Annual Meeting of The American Association for Thoracic Surgery, Boston, Mass, May 4-7, 2003.

Received for publication May 1, 2003; revisions requested Dec 12, 2003; accepted for publication Jan 28, 2004.

Address for correspondence: Alain Serraf, MD, Marie-Lannelongue Hospital, 133 Ave de la Résistance, 92350 Le PlessisRobinson, France (E-mail: aserraf@ccml. com).

J Thorac Cardiovasc Surg 2004;128:44-52 $0022-5223 / \$ 30.00$

Copyright $\odot 2004$ by The American Association for Thoracic Surgery

doi:10.1016/j.jtcvs.2004.01.040
Objective: This study was undertaken to identify potential anatomic and surgical factors creating left-sided lesions, namely recoarctation of the aorta and neoaortic regurgitation, after anatomic repair of transposition of the great arteries with ventricular septal defect and aortic coarctation.

Methods: From 1983 to September 2002, 109 survivors out of 120 patients were studied. Two-stage repair was performed in 42 patients (group A), and single-stage repair was performed in 67 (groups B and C). Before repair, the diameters of the ascending aorta and main pulmonary artery were measured. In the patients with single-stage repair, coarctation was repaired by extended end-to-end anastomosis in 35 patients (group B) and by pulmonary homograft patch augmentation in 32 patients (group C). The ventricular septal defect was closed through the pulmonary artery in 70 patients and through the right ventricle or atrium in 39 patients. The neoaorto-aortic discrepancy was treated by $\mathrm{V}$-shaped resection of the posterior sinus of Valsalva in 7 cases, pulmonary homograft patch in 32 cases, and anterior splitting of the ascending aorta in all cases. Before discharge from the hospital, neoaortic root and ascending aorta diameters and aortic regurgitation grade were recorded. Neoaortic regurgitation progression and reintervention were the end points of follow-up (97.2 \pm 61.2 months).

Results: Early and late survivals were significantly better in group $\mathrm{C}(P<.001)$. Risk factors for neoaortic regurgitation at discharge by univariate analysis were single-stage repair $(P<.05)$ and ventricular septal defect closure through the pulmonary artery $(P=.0076)$. On multivariate analysis, the latter was the only risk factor for neoaortic regurgitation at discharge and at last follow-up. Multivariate analysis showed that higher neoaortic root/ascending aorta ratio and ventricular septal defect closure through the pulmonary artery were risk factors for neoaortic regurgitation evolution at last follow-up. There were 29 reinterventions, 19 for recoarctation of the aorta and 10 for neoaortic regurgitation with or without aortic root dilatation. Group B $(P<$ $.05)$, high neoaortic root/ascending aorta ratio $(P<.01)$, and progressive neoaortic regurgitation $(P<.05)$ were risk factors for recoarctation of the aorta. Group A was a risk factor for aortic valve replacement at 10 years $(P<.05)$.

Conclusion: Neonatal single-stage repair with pulmonary homograft aortic augmentation remains the optimal approach to transposition of the great arteries with ventricular septal defect and aortic coarctation. It provides better early and late 
survivals and freedoms from left-sided lesions. Avoidance of late recoarctation of the aorta and progressive neoaortic regurgitation requires meticulous closure of the ventricular septal defect and evenly sized reconstruction of the aorta from root to distal arch.

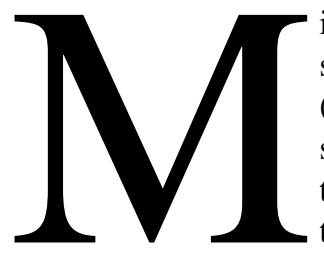

idterm and long-term follow-up results of the arterial switch operation (ASO) have demonstrated that some specific morbidity is still observed after an ASO; this consists mainly of the pulmonary outflow tract, coronary artery, and the native pulmonary valve that has become the aortic valve. ${ }^{1-7}$ The fate of the neoaortic valve has not yet been extensively studied in large populations, and the incidence of aortic regurgitation (AR) is variously assessed. ${ }^{3,8}$ AR incidence and its outcome are essential to assess because they can induce severe morbidity and shorten life expectancy. In some cases, particularly for patients with complex forms of transposition of the great arteries (TGA) associated with ventricular septal defect (VSD) and coarctation of the aorta, the incidence of postoperative neoaortic valve insufficiency is greater in this population than in the population with noncomplex TGA. In addition, recoarctation of the aorta is not an uncommon finding among these patients, although immediate postoperative aortic arch flow seems laminar. This study was undertaken to delineate whether this type of evolution, namely increased incidence of left-sided lesions, in this cohort of patients is due to the anatomic complexity of the disease or whether surgical factors are implicated. If the latter is the case, an attempt can be made to neutralize these factors.

\section{Patients and Methods}

From the beginning in 1983 of the ASO program for TGA at Marie-Lannelongue Hospital until September 2002, among 1399 patients with TGA, 120 were identified with associated VSD and coarctation. For long-term analysis of left-sided lesions, only patients who ultimately received a biventricular repair through an ASO were included in the present study. Therefore, patients who entered a 2-stage repair program but died after the palliative procedure were excluded $(n=3)$, as were 8 in-hospital deaths after ASO.

As a follow-up, it was prospectively determined that all survivors should have a yearly examination carried out by the referring cardiologist, including a clinical assessment, an electrocardiogram, and an echocardiogram with Doppler study. Catheterization with aortography or coronary arteriography was done only if deemed necessary. All these data were regularly transmitted to our center, and missing data were completed from recall of the referring cardiologist. The hospital records of all patients were reviewed for details of preoperative assessment, operative management, and hospital course. Presence and quantification of the AR was eval- uated by color Doppler imaging and graded as none (0), trivial (1) mild (2), moderate (3), or severe (4), depending on the ratio of the width of the regurgitant jet to the diameter of the high left outflow tract. ${ }^{9}$ This method was validated later in children after using it in this study. ${ }^{10}$ Because patients had only a yearly echocardiographic examination, AR when initially absent was assumed to appear just before the ultrasonographic examination that revealed it. In addition, recoarctation of the aorta was considered to have occurred when echocardiographic Doppler studies demonstrated systolic gradient across the suture line with diastolic prolongation despite an initial good result on the aortic arch. In the absence of diastolic prolongation but with systolic gradient, exercise testing was indicated when feasible to determine the appearance of a diastolic prolongation.

The observed left-sided lesions were retrospectively analyzed to validate the hypothesis that in this anomaly, the great artery anatomy is mainly associated with a size discrepancy between the ascending aorta and the main pulmonary artery (PA), with the latter being much larger than the aortic root. Furthermore, the right ventricle is generally smaller, and the coronary artery origin and course are often abnormal with anterior looping on the right ventricle. In addition, a substantial number of patients had abnormal tricuspid valve attachments on the conal septum. All these factors are considered additional risk for VSD closure through the right atrium or the right ventricle and promote VSD closure through the native PA. After ASO, continuity is established between the enlarged neoaortic root through which the VSD has been closed and a smaller distal ascending aorta and aortic arch. Although the development of a supra-aortic stenosis is an exceptional secondary occurrence, turbulence may occur and aggravate a mild AR caused by the transpulmonary VSD closure. Therefore, care during this operation to reestablish an evenly calibered ascending aorta and aortic arch should reduce the occurrence of neo-AR. In addition, if blood flow through the ascending aorta and aortic arch remains more laminar, the risk for recoarctation of the aorta should also be reduced.

\section{Data Collection}

Preoperative echocardiographic studies from all patients admitted to Marie-Lannelongue hospital for surgical treatment of TGA with VSD and coarctation were reviewed. Measurements of aortic and pulmonary annuli as well as sinotubular aortic junction and aortic arch diameters were recorded before surgery and before hospital discharge. The preoperative ratio of the main PA diameter to that of the ascending aorta was calculated and the ratios of the neoaortic root to the ascending aorta before and after anastomosis were also assessed in the postoperative period. AR seen at echocardiography was considered initially as grade 1 . During the time frame of the study, the surgical approach evolved from a 2-stage to 
TABLE 1. Causes of 12 late deaths

\begin{tabular}{lc}
\hline Cause & No. \\
\hline Residual shunt (multiple VSDs) & 3 \\
Hypoplastic right ventricle and multiple VSDs & 1 \\
Left ventricular outflow tract obstruction & 1 \\
Aortic arch hypoplasia and recoarctation & 1 \\
Pulmonary hypertension & 1 \\
Coronary-related death & 3 \\
Left pulmonary venous obstruction & 1 \\
Aortic insufficiency & 1 \\
\hline
\end{tabular}

a single-stage procedure. Therefore, relevant surgical data were recorded and 3 individual surgical groups were defined.

\section{Patients}

Group A consisted of 42 patients (38.5\%) who underwent a 2-stage surgical repair, nearly all between 1983 and 1987. An extended end-to-end anastomosis (EEEA) for coarctation associated with PA banding was performed through a left thoracotomy during the preliminary palliative stage. The complete repair, consisting of ASO and VSD closure, was performed during the second stage. The VSD was closed through a right atriotomy or the right ventricle in 25 cases $(59.5 \%)$ and through the original PA in 17 cases $(40.6 \%)$. Closure through the right atrium was always attempted. The ASO was then performed according to the standard technique in our institution.

Group B consisted of 35 patients (32.1\%) who underwent a single-stage complete repair through a median sternotomy. The aortic arch was reconstructed by EEEA. The details of surgical technique and cardiopulmonary bypass have been described previously elsewhere. ${ }^{1,12}$ The VSD was closed through a right atriotomy or the right ventricle in 10 cases $(28.6 \%)$ and through the original PA in 25 cases $(71.4 \%)$.

Group C consisted of 32 patients (29.4\%) who also underwent a single-stage complete repair through a median sternotomy, but attention was given to reestablishing an evenly sized ascending aorta and aortic arch. After resection of the coarctation shelf and ductal tissue, the medial aspect of the distal ascending aorta, the undersurface of the aortic arch, and the first centimeter of medial aspect of the proximal descending aorta were incised. Before ASO a posterior hemianastomosis was performed between the distal aortic arch and posterior wall of the proximal descending aorta. Thereafter the distal parts of ascending aorta, aortic arch, and proximal descending aorta were augmented with a pulmonary homograft patch for treating neoaorto-aortic size mismatch and aortic arch hypoplasia. Recently, the aortic arch reconstruction has been performed without circulatory arrest by selective cerebral perfusion. The closure of the VSD was achieved through the original PA in 28 cases $(87.5 \%)$ and through the right ventricle or a right atriotomy in 4 cases $(12.5 \%)$.

Another surgical technique to treat neoaorto-aortic noncongruence that was applied to patients with lower ratio of the main PA diameter to that of the ascending aorta was achieved by V-shaped resection of the posterior new aortic sinus of Valsalva in 7 patients (4 in group A and 3 in group B). In these patients the coarctation was repaired by EEEA. In addition, the vast majority of patients underwent reimplantation of the right coronary button above the aortic suture line after anterior splitting of the ascending aorta, which also made it possible to restore a more evenly sized aorta.

\section{Follow-up}

The information for this study was gathered by retrospective review of patients' records during the summer of 2002. Mean follow-up was $97.2 \pm 61.2$ months. The occurrence of left-sided lesions was determined from a yearly echocardiographic study. AR was considered to be already present when graded as trivial (grade 1). Progressive AR was considered when the grade of the regurgitant jet increased. All reinterventions, whether percutaneous or surgical for either recoarctation of the aorta or AR, were identified and considered as the end point for the follow-up of this study. Eight patients with in-hospital deaths were excluded from leftsided lesion follow-up.

\section{Statistical Analysis}

All measured values are expressed as mean \pm SD. Statistical comparisons for identifying the risk factors for left-sided lesions were performed by the Student $t$ test and the $\chi^{2}$ test for continuous and categorical variables, respectively. Multivariate analysis was performed by logistic regression model. The relationship between neoaorta/ascending aorta diameter ratio and progression of AR was evaluated by the Mann-Whitney nonparametric $U$ test. Timerelated freedom from reintervention (recoarctation of the aorta and aortic valve replacement [AVR]) and survival were described by actuarial methods, and the differences among groups were calculated by the log-rank test. The parametric multiphase hazard function was also applied to evaluate the probability of unfavorable outcome event. Ratios are expressed with $70 \%$ confidence interval (CI).

\section{Results \\ Mortality}

None of the patients had pulmonary valve insufficiency before anatomic repair. Total early mortality, including the patients of group A who died after the first stage of the operation, was $9.16 \%$ (70\% CI 6.2\%-12.7\%). Early mortality was $6.8 \%(70 \%$ CI $4.2 \%-10.1 \%)$ after biventricular repair $(n=8)$. It was $12.5 \%$ in group $A(n=6), 10.2 \%$ in group B $(n=4)$, and $3 \%$ in group C $(n=1)$, significantly lower in group $\mathrm{C}$ than in groups $\mathrm{A}$ and $\mathrm{B}(P<.001)$. No patient was unavailable for follow-up. Among the survivors of the initial hospital stay, 12 late deaths (Table 1) occurred (11\%, $70 \%$ CI 7.7\%-15\%). Overall mortalities, including the in-hospital, interim, and late mortalities, were $27.1 \%$ in group A, $20.5 \%$ in group B ( $P=.03$ vs group A), and $6.1 \%$ in group $\mathrm{C}(P<.001$ vs groups $\mathrm{A}$ and $\mathrm{B})$. Overall actuarial survivals at 1 and 10 years were $90 \% \pm 2.8 \%$ and $67.4 \% \pm$ $5 \%$, respectively (Figure 1). Figure 2 depicts the actuarial survivals in the 3 different groups.

\section{General Characteristics of Patients}

The PA/ascending aorta ratio was significantly higher $(P<$ $.0001)$ in patients with single-stage repair $(1.9 \pm 0.2)$ than 


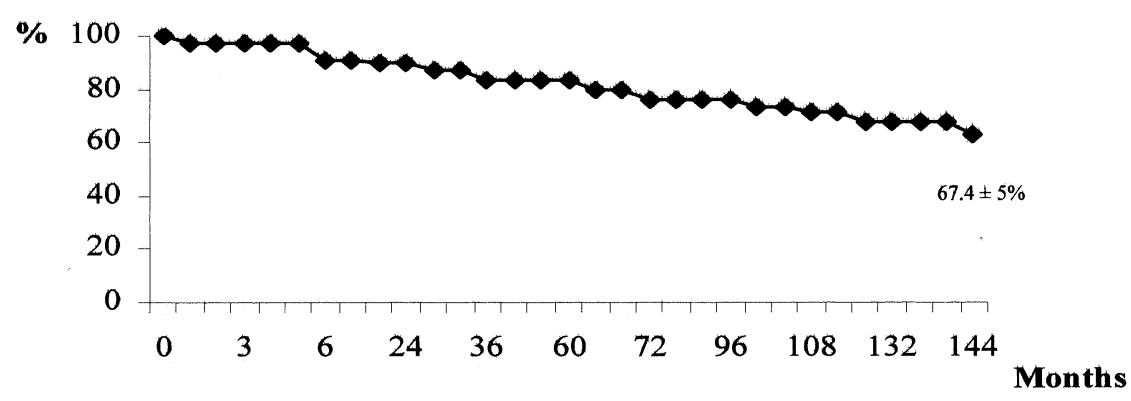

Figure 1. Overall actuarial survival of patients with TGA, VSD, and coarctation.

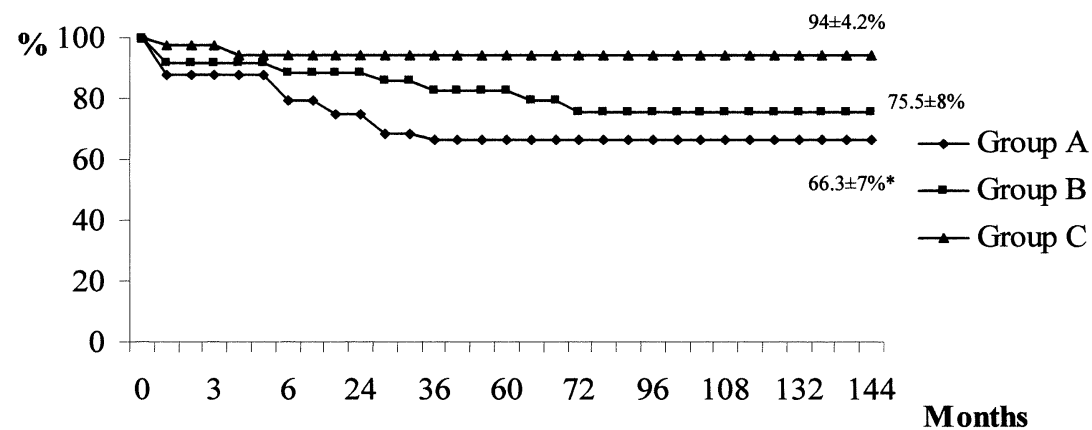

Figure 2. Actuarial survival according to surgical group. Asterisk indicates $\boldsymbol{P}<.05$ by log-rank test.

in patients with 2-stage repair $(1.3 \pm 0.1)$. Furthermore, the rate of VSD closure through the original PA was higher among patients with single-stage repair $(P<.01)$. The correction of neoaorto-aortic discrepancy was achieved by patch aortoplasty or V-shaped resection of noncoronary sinus of the neoaortic root and was assessed by ratio of the neoaortic root to the ascending aorta. It was $1.15 \pm 0.2$ in group $\mathrm{A}, 1.3 \pm 0.2$ in group $\mathrm{B}$, and $1.05 \pm 0.1$ in group $\mathrm{C}$ $(P<.001$ vs groups $\mathrm{A}$ and $\mathrm{B})$.

\section{Neo-AR}

At discharge. AR was present in 52 patients $(47.7 \%$, $70 \% \mathrm{CI} 42.2 \%-53.1 \%)$. It was trivial in $44(84.6 \%)$ and grade $2 / 4$ or above in $8(15.4 \%)$. Among those patients, 15 (28.8\%) were in group A, 19 (36.5\%) were in group B, and 18 (34.6\%) were in group C. Five, 1 , and 2 patients had grade $2 / 4$ or greater $\mathrm{AR}$ in groups $\mathrm{A}, \mathrm{B}$, and $\mathrm{C}$, respectively $(P=.25)$. The total prevalence of AR among patients who underwent a single-stage approach $(55.2 \%)$ was significantly $(P<.05)$ higher than that among patients who underwent a 2-stage approach, but the difference was not statistically significant if only grade $2 / 4$ or greater AR was considered.

The occurrence of AR was significantly related $(P=$ .0076) to the approach for VSD closure. Among the 70 patients whose VSD was closed through the native PA, 40 had postoperative AR. However, when only AR grade 2/4 or greater was considered, the route for VSD closure was not a risk factor.
Pulmonary homograft patch aortoplasty to treat coarctation, aortic arch hypoplasia, and decreasing neoaortic root/ ascending aorta discrepancy was performed in 32 cases. AR occurred in 20 of these, which was significantly $(P<.05)$ higher than the occurrence of AR among patients who did not undergo patch aortoplasty . However, when only grade 2/4 or greater AR was considered, these differences were not significant.

Because restoration of an evenly calibered ascending aorta, aortic arch, and isthmus was found in patients from the 3 different surgical groups, occurrence of postoperative AR was also analyzed through the aortic root/ascending aorta ratio. This ratio was found not to be a risk factor for postoperative AR.

Multivariate analysis by logistic regression model showed that VSD closure through the native PA was the single risk factors for neoaortic valve insufficiency at discharge from the hospital $(P<.01)$.

At last follow-up. AR was present in 46 patients (42.2\%, $70 \%$ CI 36.8\%-47.6\%). It was trivial in 18 patients and grade $2 / 4$ or greater in 28 patients. Fifteen patients with AR (32.6\%) were in group A, 16 (34.8\%) were in group B, and 15 (32.6\%) were in group C. Ten, 12, and 6 patients had more severe AR (grade $2 / 4$ or more) in groups A, B, and C, respectively $(P=.33)$. Furthermore, there was no statistical difference in the incidence of AR according to whether repair was performed as a 2-stage or single-stage approach. 
Again, the occurrence of AR at last follow-up was highly related $(P<.0001)$ to the approach for VSD closure. There were 39 patients with AR whose VSD was closed through the original PA. In contrast with the occurrence of AR at discharge, when only grade $2 / 4$ or greater was considered, the route for VSD closure through the original PA remained a significant risk factor $(P=.0037)$.

Evolution. AR evolution was evaluated during follow-up and defined as any variation of neo-AR. Thirty one patients demonstrated an upgrade evolution of the AR (28.4\%, 70\% CI 23.6\%-33.6\%). Ten of these patients (23.8\%) belonged to group A, 14 patients (40\%) belonged to group $\mathrm{B}$, and 7 patients $(21.8 \%)$ belonged to group $\mathrm{C}(P$ not significant). The total number of patients with AR evolution who underwent a single-stage approach $(31.35 \%)$ was not significantly different from those who underwent a 2-stage approach (33.3\%). The occurrence of AR upgrade was highly $(P=.0001)$ related to the VSD closure approach. AR upgrade occurred in 8 patients $(25 \%)$ with patch aortoplasty, which was less common ( $P$ not significant) than AR evolution among patients without patch aortoplasty (30\%). In 15 patients AR grade did not show any evolution throughout the duration of follow-up.

In addition to the approach for VSD closure through the native PA, the postoperative aortic root/ascending aorta ratio was highly correlated with an aggravation of any previous AR (Mann-Whitney test, $P=.02$ ). Recoarctation was not a risk factor for AR evolution.

In 13 patients a trivial AR developed that was not present at discharge from the hospital, and in 19 cases trivial AR present at discharge disappeared during follow-up. In these patients, the ratio of the neoaortic root to the ascending aorta was the only factor associated with the appearance or disappearance of $\mathrm{AR}(P<.0001)$.

Multivariate analysis model showed that both the approach for VSD closure through the original PA and higher postoperative ratio of the neoaortic root to the ascending aorta were risk factors for neo-AR evolution at last follow-up $(P<.0001)$.

\section{Recoarctation}

Recoarctation of the aorta occurred in 19 patients: 4 in group A, 12 in group B, and 3 in group C. All underwent an intervention for recoarctation. The group B approach was significantly associated with higher rate of recoarctation $(P$ $<.05)$. Aortic root/ascending aorta ratio was also a significant risk factor for recoarctation of the aorta $(P<.001)$, independently of the presence of any AR. However, progressive AR was significantly associated with recoarctation $(P<.05)$.

\section{Other Left-Sided Lesions}

During the follow-up, 2 patients had myocardial infarction at 3 and 6 months after the operation. One died before any attempt at coronary revascularization, and the other had a heart transplantation but also died of rejection. A third patient died suddenly 3 months after the operation, probably of a coronary artery event. Subaortic stenosis was a matter of concern in 2 patients: 1 had sudden death and the other is undergoing close follow-up.

\section{Reintervention}

Among the 109 survivors, of the 50 patients who underwent reoperations, 29 had reintervention for left-sided lesions, namely progressive $\mathrm{AR}$, recoarctation of the aorta, or both. Of these, AVR consisted of simple valve replacement for 4 patients, modified Bentall procedure for 3 patients, and modified Bentall procedure with aortic arch repair for 3 patients. No deaths occurred after AVR. Nineteen patients underwent reintervention for recoarctation either by surgical repair $(\mathrm{n}=9$, with 1 death) or percutaneous balloon dilatation $(\mathrm{n}=10)$. Actuarial freedoms from reintervention for left-sided lesions were $95.2 \% \pm 0.02 \%, 84.3 \% \pm 0.04 \%$, and $58.7 \% \pm 0.08 \%$ at 1,5 , and 10 years, respectively. These rates in the 3 different groups are depicted in Figure 3 for recoarctation and in Figure 4 for AVR. The 10-year freedom from AVR in group C ( $96 \%)$ was significantly $(P$ $<.05)$ higher than that in group A $(40 \%)$. The mean period between ASO and AVR was $83 \pm 43$ months.

\section{Discussion}

Since the beginning of our program of anatomic correction of TGA, neonatal single-stage repair of TGA, VSD, and aortic arch obstruction has increasingly become the procedure of choice and has yielded low mortality and morbidity. In addition to the physiologic advantages of early anatomic repair, the cumulative risk of morbidity from the 2-stage management is avoided. ${ }^{11}$ The presence of associated multiple VSDs remains the principal contraindication for single-stage repair. However, as time elapsed after the repair, an increased occurrence of left-sided lesions, namely AR and recoarctation, was observed in this cohort of patients. Because the rate of late AR occurrence was higher in this group than among other patients who also underwent anatomic repair for various forms of TGA, the question was whether this late morbidity was related to anatomic factors or surgical technique.

In analyzing our entire series of 1265 survivors after an ASO, the occurrence of AR was found to be significantly higher in the group of patients who had TGA with VSD and coarctation. In that group the occurrence of AR was $25.7 \%$, compared with $2 \%$ in the remaining population of patients with TGA (8\% among patients with TGA and VSD and 2/1000 among patients with TGA and intact ventricular septum). The surgical technique for coronary relocation was therefore addressed to find out whether it could cause AR. The current surgical technique for coronary relocation is roughly applied to all type of TGA regardless of the com- 


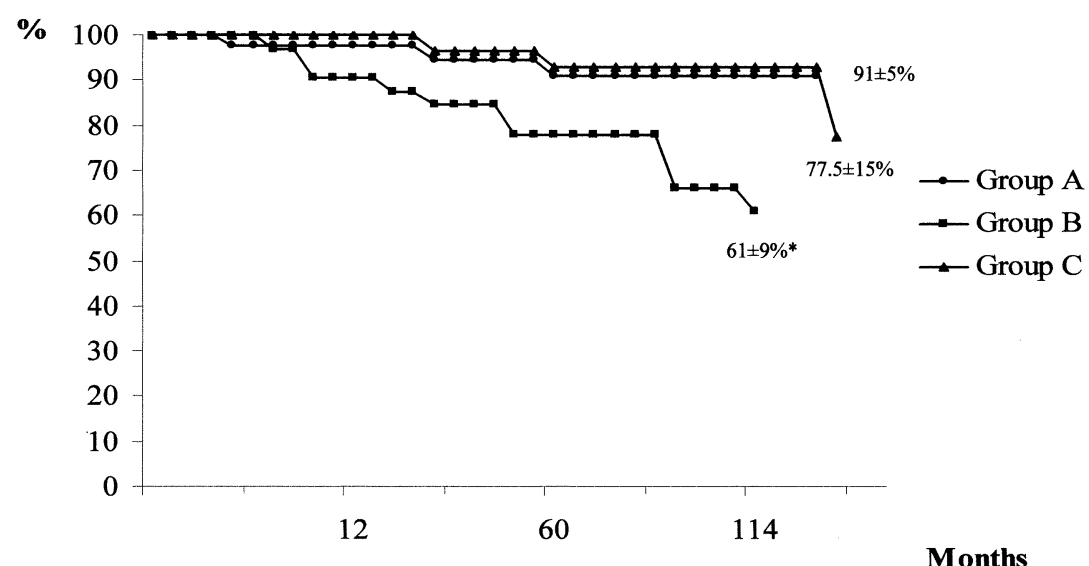

Figure 3. Freedom from recoarctation according to surgical group. Asterisk indicates $P<.05$ by log-rank test.

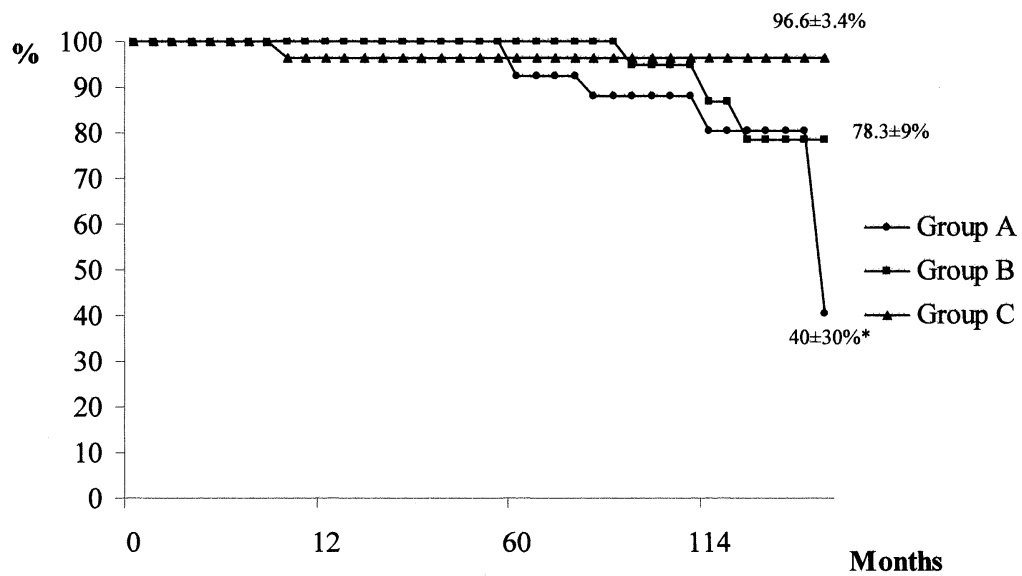

Figure 4. Freedom from AVR according to surgical group. Asterisk indicates $P<.05$ by log-rank test.

plexity of associated intracardiac and extracardiac malformation and the type of coronary patterns. The left coronary ostium is reimplanted in a low position through a trapdoor incision in the adjacent sinus of Valsalva, and the right coronary ostium is reimplanted in a high position above the aortic suture line. Because the same technique was applied to all patients with TGA, it was determined that coronary relocation was not involved in the postoperative development of AR.

In the group of patients who had TGA with VSD and coarctation, one of the main anatomic factors consisted of the discrepancy between diameters of the native pulmonary root (neoaorta) and ascending aorta and the constant presence of aortic arch hypoplasia. In addition, the anterior displacement of the subpulmonary conus can result in a situation in which the upper edge of the VSD is mainly constituted by one or two neoaortic cusps. This anatomic particularity could interfere with surgical difficulties, resulting in left-sided lesions. The following hypothesis was therefore advanced: Because the right ventricle is generally smaller and coronary artery origin and course are often abnormal with anterior looping on the right ventricle, ${ }^{13,14}$ VSD closure through the right atrium or the right ventricle might be at risk; these factors encourage VSD closure through the native PA when not feasible through another approach. After ASO, aortic continuity is established between the enlarged neoaortic root and a smaller distal ascending aorta and aortic arch. This anatomic configuration could lead to turbulent blood flow through the new proximal aorta, which might aggravate a mild AR because of the transpulmonary VSD closure. In addition, if blood flow through the ascending aorta and aortic arch remains more laminar, the risk for recoarctation should also be reduced.

\section{Neo-AR}

Although, the incidence of AR at discharge was $47.7 \%$, the degree of AR was trivial in most cases (84.6\%). Univariate analysis showed that transpulmonary VSD closure, singlestage repair, and use of pulmonary homograft patch aortoplasty were significant risk factors for AR. However, mul- 
tivariate analysis demonstrated that transpulmonary VSD closure was the unique significant risk factor for subsequent AR. Although this approach is considered easier and faster, additional precaution is needed to keep from damaging the neoaortic valve. For this reason, the surgical technique has been modified when the transpulmonary route is considered. The upper edge of the VSD, which corresponds to the subpulmonary conus, is generally masked by the overriding of the pulmonary annulus; it can, however, be unfolded and pulled out by some $U$ traction sutures across the outer ventricular wall, below the neoaortic annulus, and emerging in the conus. These sutures should be applied after coronary button harvesting and dissection in order to avoid coronary injury. In this manner the VSD edges are well exposed, and it can be closed carefully without any damage through the neoaortic valve. This approach has been applied to recent patients without any trace of postoperative AR. Among patients who underwent a 2-stage repair the rate of VSD closure through PA was significantly lower than among those with single-stage repair, because coarctation repair associated with PA banding allowed growth of the right ventricle and atrium. Therefore, the neoaortic valve was less at risk for intraoperative injury.

Between discharge and the last visit, the initial postoperative AR disappeared in 19 patients. On the other hand, in 13 patients discharged without evidence of AR, development of AR was documented. At last follow-up, 46 patients had AR, $61 \%$ grade 2 or above. Statistical analysis revealed that transpulmonary VSD closure remained a significant risk factor for AR in all periods. Independently from other factors, the discrepancy between the new aortic root and ascending aorta, as expressed by the ratio of the neoaortic root to the ascending aorta, was highly associated with the secondary appearance or disappearance of AR, as well as the upgrade evolution of a previous AR. Among patients for whom the aortoaortic discrepancy was intraoperatively managed either by homograft patch or by V-shaped resection technique, the evolution of AR grade at follow-up was significantly less frequent than among the remaining patients. This suggests the benefits of surgical techniques that normalize the aortic root/ascending aorta ratio. Interestingly enough, patients who underwent 2-stage repair had a lower $\mathrm{PA} /$ ascending aorta ratio, suggesting that the first palliative procedure allows growth not only of the right ventricle but also of the ascending aorta. In these patients the postoperative aortic root/ascending aorta ratio was also significantly lower than in patients who underwent single-stage repair without patch aortoplasty.

Among the 31 patients with AR upgrade, 10 patients required AVR with or without aortic root replacement by the modified Bentall procedure, with a median delay of 7 years as a late event. In all of these patients some degree of retraction and fibrosis in the valve tissue adjacent to the prosthetic patch of VSD closure was found.

\section{Recoarctation}

Recoarctation was the principal cause of reintervention. Ten patients underwent balloon angioplasty; 9 others required surgery. Although we as well as others ${ }^{15,16}$ have shown the feasibility of EEEA by means of an anterior approach, group B (single stage and EEEA) patients were more likely to develop recoarctation of the aorta. Extensive mobilization of the descending aorta through a median sternotomy is more difficult and results in performance of EEEA under some degree of traction. This could be the cause of more frequent recoarctation in this group of patients. Some published series ${ }^{15,17,18}$ have suggested the use of patch aortoplasty to treat aortic coarctation and aortic arch hypoplasia with low rates of recurrence. It diminishes the anastomotic tension and also provides an evenly calibered aortic arch, thus reducing the risk of recoarctation and constructing an evenly sized aorta with a decreasing ratio of the neoaortic root to the ascending aorta.

A higher aortic root/ascending aorta ratio was statistically significantly related to recoarctation of the aorta. This was probably due either to the neglected severity of the aortic arch hypoplasia, which was not treated during the first operation, or to the absence of the laminar blood flow across the aortic arch. In addition a progressive AR was also associated with a higher rate of recoarctation. These data suggest the importance of aortic blood flow for remodeling of the aorta. Indeed, normal flow through the vascular system has been shown to maintain adequate remodeling of the vessel, ${ }^{18}$ and one can imagine that in cases in which aortic anatomy has not been respected, in addition to a regurgitant jet to the left ventricle through an AR, the resultant flow crossing the aortic isthmus should be abnormal and promote the constriction of previous sutures.

The high number of modified Bentall operations in this series confirms the presence of aortic root dilatation associated with AR. Several reports ${ }^{19-22}$ have demonstrated an increased incidence of AR in association with disproportionate dilatation of the neoaortic root after ASO without analyzing the implication of surgical factors. The tendency for neoaortic root dilatation to progress with time after repair and nearly normal growth of the aortic anastomosis underlines the importance of treating aortic root/ascending aorta discrepancy during ASO. Also, Jenkins and colleagues ${ }^{23}$ analyzed the fate of the pulmonary valve in the systemic circulation and concluded that structural factors could be the cause of late regurgitation. In this work, it was demonstrated that beyond these factors inadequate surgical technique can also seriously interfere with the late outcome of these patients. PA banding has been addressed as a cause of late AR because of pulmonary root dilatation, elevated PA pressure, and increased flow through the pulmonary 
valve $^{24}$; however, statistical analysis did not discriminate this procedure as a risk factor, probably because the time between the first and second procedures did not exceed 6 months.

\section{Conclusion}

In conclusion, neonatal single-stage repair with pulmonary homograft aortic arch augmentation remains the optimal approach for patients who have TGA with VSD and aortic arch obstruction. It provides better early and late survivals and freedoms from left-sided lesions. However, this complex procedure should take into account the long-term morbidity associated with left-sided lesions. Such lesions can be avoided by closure of the VSD through the right atrium or right ventricle. When these approaches are considered to carry a too high risk, transpulmonary VSD closure remains a valuable approach provided that the surgical technique can expose all the edges of the VSD, particularly the upper edge in close relation with the aortic valve, and provided that there is restoration of an evenly calibered aorta from neoaortic root to isthmus. This can be achieved by aortic patch augmentation, $\mathrm{V}$-shaped resection of the noncoronary sinus, or even a 2 -stage repair.

\section{References}

1. Wernovsky G, Mayer JE, Jonas RA, Hanley FL, Blackstone EH, Kirklin JW, et al. Factors influencing early and late outcome of the arterial switch operation for transposition of the great arteries. $J$ Thorac Cardiovasc Surg. 1995;109:289-302.

2. Serraf A, Roux D, Lacour-Gayet F, Touchot A, Bruniaux J, Sousa-Uva M, Planché C. Reoperation after the arterial switch operation for transposition of the great arteries. J Thorac Cardiovasc Surg. 1995; 110:892-9.

3. Haas F, Wottke M, Poppert H, Meisner H. Long-term survival and functional follow-up in patients after the arterial switch operation. Ann Thorac Surg. 1999;68:1692-7.

4. Daebritz SH, Nollert G, Sachweh JS, Engelhardt W, von Bernuth G, Messmer BJ. Anatomical risk factors for mortality and cardiac morbidity after arterial switch operation. Ann Thorac Surg. 2000;69: 1880-6.

5. Brown JW, Park HJ, Turrentine MW. Arterial switch operation: factors impacting survival in the current era. Ann Thorac Surg. 2001;71: 1978-84.

6. Prêtre R, Tamisier D, Bonhoeffer P, Mauriat P, Pouard P, Sidi D, et al. Results of the arterial switch operation in neonates with transposed great arteries. Lancet. 2001;357:1826-30.

7. Losay J, Touchot A, Serraf A, Litvinova A, Lambert V, Piot JD, et al. Late outcome after arterial switch operation for transposition of the great arteries. Circulation. 2001;104(12 Suppl 1):I121-6.

8. von Bernuth GV. 25 years after the first arterial switch procedure: mid-term results. Thorac Cardiovasc Surg. 2000;48:228-32.

9. Perry GJ, Helmcke F, Nanda NC, Byard C, Soto B. Evaluation of aortic insufficiency by Doppler color flow mapping. J Am Coll Cardiol. 1987;9:952-9.

10. Tani LY, Minich LL, Day RW, Orsmond GS, Shaddy RE. Doppler evaluation of aortic regurgitation in children. Am J Cardiol. 1997;80: 927-31.

11. Planché C, Serraf A, Comas JV, Lacour-Gayet F, Bruniaux J, Touchot A. Anatomic repair of transposition of great arteries with ventricular septal defect and aortic arch obstruction. One-stage versus two-stage procedure. J Thorac Cardiovasc Surg. 1993;105:925-33.

12. Serraf A, Lacour-Gayet F, Bruniaux J, Touchot A, Losay J, Comas J, et al. Anatomic correction of transposition of the great arteries in neonates. J Am Coll Cardiol. 1993;22:193-200.

13. Milanesi O, Thiene G, Bini RM, Pellegrino PA. Complete transposition of the great arteries with coarctation of aorta. Br Heart J. 1982; 48:566-71.

14. Moene RJ, Ottenkamp J, Oppenheimer-Dekker A, Bartelings MM. Transposition of the great arteries and narrowing of the aortic arch. Emphasis on right ventricular characteristics. Br Heart J. 1985;53:58-63.

15. Vitullo DA, Deleon SY, Graham LC, Eidem BW, Roughneen PT, Javorski JJ, et al. Extended end to end repair and enlargement of the entire arch in complex coarctation. Ann Thorac Surg. 1999;67:528-33.

16. Lacour-Gayet F, Serraf A, Galletti L, Bruniaux J, Belli E, Piot D, et al. Biventricular repair of conotruncal anomalies associated with aortic arch obstruction. Circulation. 1997;96(9 Suppl):II328-34.

17. Tchervenkov CT, Tahta SA, Jutras L, Beland MJ. Single-stage repair of aortic arch obstruction and associated intracardiac defects with pulmonary homograft patch aortoplasty. J Thorac Cardiovasc Surg. 1998; 75:897-904.

18. Roussin R, Belli E, Lacour-Gayet F, Godart F, Rey C, Bruniaux J, et al. Aortic arch reconstruction with pulmonary autograft patch aortoplasty. J Thorac Cardiovasc Surg. 2002;123:443-50.

19. Song JK, Kang DH, Lim TH, Song MG, Kim JJ, Park SW, et al. Different remodeling of descending thoracic aorta after acute event in aortic intramural hemorrhage versus aortic dissection. Am J Cardiol. 1999;83:937-41.

20. Martin RP, Ettedgui JA, Qureshi SA, Gibbs JL, Baker EJ, RadleySmith R, et al. A quantitative evaluation of aortic regurgitation after anatomic correction of transposition of the great arteries. J Am Coll Cardiol. 1988;12:1281-4.

21. Hourihan M, Colan SD, Wernovsky G, Maheswari U, Mayer JE, Sanders SP. Growth of the aortic anastomosis, annulus, and root after the arterial switch procedure performed in infancy. Circulation. 1993; 88:615-20.

22. Hutter PA, Thomeer BJ, Jansen P, Hitchcock JF, Faber JA, Meijboom EJ, et al. Fate of the aortic root after arterial switch operation. Eur J Cardiothorac Surg. 2001;20:82-8.

23. Jenkins KJ, Hanley FL, Colan SD, Mayer JE, Castaneda AR, Wernovsky G. Function of the anatomic pulmonary valve in the systemic circulation. Circulation. 1991;84(5 Suppl):III173-9.

24. Sievers HH, Lange PE, Arensman FW, Radley-Smith R, Yacoub MH, Harms D, et al. Influence of two-stage anatomic correction on size and distensibility of the anatomic pulmonary/functional aortic root in patients with simple transposition of the great arteries. Circulation. 1984;70:202-8.

\section{Discussion}

Dr Christo I. Tchervenkov (Montreal, Quebec, Canada). I congratulate the group from Marie-Lannelongue for their outstanding contributions through the years to the surgical treatment of this most difficult group of patients. TGA, VSD, and aortic arch obstruction constitute a complex congenital heart malformation that unfortunately needs to be tackled in the neonatal period.

The traditional 2-stage approach has been associated with a high mortality. Because of this, since my return from Boston in the late 1980s our approach at the Montreal Children's Hospital has been to perform a single-stage ASO, VSD closure, and concomitant aortic arch repair with pulmonary homograft patch aortoplasty. This approach was not based on any data, and I am particularly pleased to see that the Paris group is validating our approach for the last 14 years. Professor Planché, at the 1992 meeting of The American Association for Thoracic Surgery in Los Angeles, presented conclusive evidence regarding the superiority of the single-stage approach to the 2-stage approach, and Dr Serraf has now presented further evidence that perhaps the optimal technique of aortic arch repair is the pulmonary homograft patch aortoplasty. 
The pulmonary homograft patch aortoplasty fulfills three important objectives. It achieves an aortic arch of adequate size regardless of the original diameter, it completely corrects the often marked discrepancy between the proximal neoaorta and the distal aorta, and it eliminates the tension of the two circumferential anastomoses that are associated with the extended end-to-end technique.

What is particularly disturbing in the Paris series in the longterm follow-up is the high incidence of left-sided problems and the greater than $25 \%$ incidence of reintervention, particularly for AR and aortic arch obstruction. This may be due to factors associated with the intrinsic complexity of the disease or may be associated with the surgical techniques of correction. In Montreal since 1988 we have consistently used a single-stage approach with a pulmonary homograft patch aortoplasty with a $4 \%$ operative mortality. What is reassuring in our technique is that reintervention was only seen in 1 patient who underwent a balloon angioplasty for recurrent coarctation, and no patient had reintervention for AR.

I have the following questions. Because we still have to be reassured regarding the ongoing superiority of the single-stage approach, could you please, Dr Serraf, tell us what were the overall mortalities in the 3 groups, including the mortality after initial palliation and before repair in group $\mathrm{A}$ ? What were the reintervention rates in the individual groups, and do the data perhaps support the superiority of the technique used in group C? Was there any significant difference in mortality or reintervention rate between groups $\mathrm{B}$ and $\mathrm{C}$ ?

In addition to these two left-sided problems, there may be additional left-sided problems either early or late. What were the incidences of coronary artery occlusion or stenosis and of subaortic stenosis after repair, taking into account that in most of your recent patients you are closing the VSD through the neoaortic valve? Was the occurrence of subaortic stenosis related to the technique of VSD closure? Finally, could you again reiterate your opinion on the optimal technique for aortic arch repair and VSD closure on the basis of your experience in Paris?

I congratulate you and your group for your contributions to the surgical treatment of this group of patients.

Dr Serraf. The goal of this work was not to redemonstrate any superiority of the single- versus the 2-stage approach. However, if you need to be reassured, the overall mortalities, including the interim mortality for group A, were as follows: 13 deaths in group A, 8 in group B, and 2 in group C. Although the differences did not reach statistical significance, when analyzing the long-term survival, we were able to find a better survival for single-stage repair and certainly for group C. Finally, the superiority of the singlestage approach mainly relies on the fact that the patient theoretically does not require any major reoperation.

The major reintervention rate was found in group B. In terms of reintervention on the aortic valve, there were no differences between groups. Extended EEEA to repair the aortic arch and without a pulmonary homograft to augment the aortic arch through midline was a serious risk factor for reoperation.

Two patients died during the long-term follow-up from myocardial infarction and thus coronary artery stenosis. One had this diagnosed before death and underwent transplantation but unfortunately died some months after that.
When we looked back at the data, we found 7 patients in whom subaortic stenosis developed, and among those patients 4 died during the follow-up even before any attempt at reintervention. So the important message is that subaortic stenosis is a serious issue in this cohort of patients and should be taken into account quite early. We do not believe that this was related to the technique of VSD closure; rather, it was due mainly to the malalignment of the VSD and therefore disease related.

In my opinion the best technique still remains the single-stage approach with patch augmentation of the entire aortic arch. For VSD closure, we believe this is easier and faster through the PA. However, with these data and results in mind, we have modified the approach, and by now we just expose differently the edge of the VSD. After harvesting the coronary artery, we pass a suture through the left ventricular wall, and this suture emerges in the subpulmonary conus and can unfold the upper edge of the VSD. The patch can be sewn easily particularly in this area at risk away from aortic valve apparatus.

Sir Magdi H. Yacoub (London, United Kingdom). My question relates to AR and the need for aortic valve replacement. Do you think that anatomic abnormalities of the aortic valve contributed to this, in view of the fact that patients with coarctation commonly have abnormalities of the aortic valve? Have you compared your results in this group of patients with those of patients with TGA and VSD in the absence of coarctation of the aorta?

Dr Serraf. A previous unpublished work from our institution analyzed the outcome of the aortic valve after the ASO. We found that it was this group who had the higher rate of long-term AR. In the group with TGA and VSD, for example, where we also closed the VSD through the PA, we didn't have a much higher rate of aortic valve morbidity and reoperation. The difference between the groups of patients was statistically significant. Also, we do not believe that this was due to the pulmonary valve itself but probably to the surgical technique that we used during this time.

Dr Christian P. R. Brizard (Melbourne, Australia). Have you found any difference in the rate of AR according to the way you reconstructed the aortic root and whether there is a disruption at the time of the repair of the sinotubular junction either by a wedge resection of the posterior sinus or by the reimplantation of the coronary arteries?

Dr Serraf. We did analyze this issue. Resection of the noncoronary sinus was never associated with aortic valve insufficiency. However, this technique was applied only in 2-stage operations because we were reluctant to perform it in neonates. Coronary reimplantation generally follows the same rules regardless of the underlying form of TGA. We like to reinsert the left coronary ostia below the sinotubular junction through a trapdoor incision and the right coronary ostia above the sinotubular junction. Almost $98 \%$ of patients undergoing ASO received this type of reimplantation, and only those who had TGA with VSD and coarctation demonstrated so high a rate of aortic valve problems. The technique of coronary reimplantation was not a risk factor for aortic valve insufficiency in the entire series of patients undergoing ASO at our institution. Actually, the real relationship between the occurrence and evolution of AR was the quality of the reconstruction of the ascending aorta. 